\title{
How one experiences and embodies compassionate mind training influences its
}

\section{effectiveness}

\author{
Marcela Matos ${ }^{1}$, Joana Duarte $^{1}$, Cristiana Duarte $^{1}$, Paul Gilbert $^{2}$ \\ and José Pinto-Gouveia ${ }^{1}$
}

${ }^{1}$ Cognitive and Behavioural Research Centre (CINEICC), University of Coimbra

${ }^{2}$ Centre for Compassion Research and Training, University of Derby, College of Health and Social Care Research Centre, Derby, UK, DE22 1GB

Correspondence concerning this manuscript should be addressed to:

Marcela Matos

CINEICC, Faculdade de Psicologia e Ciências da Educação, Universidade de Coimbra Rua do Colégio Novo, Apartado 6153

3001-802 Coimbra, Portugal

Telephone: (+351) 239851450

Email: marcela.s.matos@gmail.com

\section{Compliance with Ethical Standards}

Conflict of Interest: The authors declare that they have no conflict of interest.

Funding: This study was funded by the first author (Marcela Matos) PostDoctoral grant (SFRH/BPD/84185/2012), sponsored by the Portuguese Foundation for Science and Technology (FCT).

This work was also part funded by the Compassionate Mind Foundation charity (www.compassionatemind.co.uk). 


\section{Abstract}

This paper explores indicators of practice quality of a brief compassion mind training (CMT) intervention and their impact on the development of an inner sense of one's compassionate self (CS) and a range of self-report measures. Participants were randomly assigned to one of two conditions: Compassionate Mind Training (CMT; $n=77)$ and Wait-List Control. Participants in the CMT condition practiced a range of CMT practices during two weeks. Each week, participants completed a feedback questionnaire, measuring practice frequency, helpfulness and embodiment of the practices in everyday life. Self-report measures of compassion, positive affect, shame, self-criticism, fears of compassion, and psychopathological symptoms were also completed at pre and post. Practice frequency was associated with the frequency and easiness of embodiment of the CS. Perceived helpfulness of the practices was related to greater embodiment of the CS and to increases in compassion, reassured self, relaxed and safe affect, and decreases in self-criticism. The embodiment variables of the CS were associated with higher compassion for the self, for others and from others, and with improvements in reassured self, safe affect and compassionate goals. Embodiment of the CS and perceived helpfulness of the practices predicted compassion for the self and experience of compassion from others at post-intervention. Perceiving compassion cultivation practices as helpful and being able to embody the CS in everyday life are key to foster self-compassion and the experience of receiving compassion from others, as well as to promote feelings of safeness, contentment and calmness.

Key words: Compassion; Practice quality; Practice frequency; Practice helpfulness; Embodiment; Compassion Mind Training 


\section{Introduction}

There is growing research to show that adopting prosocial, caring and compassionate attitudes and behaviours to oneself and others has a range of physical and mental benefits (Hofmann, Grossman, \& Hinton, 2011; Jazaieri, et al., 2013; Keltner, Kogan, Piff, \& Saturn, 2014; Pace et al., 2009; Ricard, 2015; Schanche, Stiles, McCullough, Svartberg, \& Nielsen, 2011; Singer \& Bolz, 2012). Fredrickson, Cohn, Coffey, Pek and Finkel (2008) found that six group sessions, with home practice based on a CD of loving-kindness meditations, increased positive emotions, mindfulness, feelings of purpose in life, social support, and decreased illness symptoms. Pace, Negi, Adame et al., (2009) found that 6 weeks of compassion meditation improved immune function, neuroendocrine and behavioural responses to stress. Compassion training has also been shown to increase physiological activation of brain areas associated with positive affect and affiliation (Weng, Fox, Shackman et al, 2013), including in response to emotionally distressing stimuli (Klimecki, Leiberg, Lamm, \& Singer, 2012), and it also impacts heart rate variability (Kirby, Doty, Petrocchi \& Gilbert 2017).

Currently, there is a range of compassion training procedures each with some evidence of effectiveness (Kirby, 2016). One approach called compassion focused therapy (CFT) was developed as a psychotherapy for high shame and self-critical people with complex mental health problems (Gilbert, 2000, 2010) for which there is good evidence of effectiveness (Kirby, 2016; Leaviss, \& Uttley, 2014). The focus of this therapy is to cultivate and attune with care-focused motives, intentions and friendly affiliative emotions to act as inner supports and antidotes to hostile and fearful ones and shame based and traumatic memories (Gilbert, 2000; 2010). CFT has two aspects. One is 'therapy' focused related to formulation, development of the therapeutic relationship, and strategies of intervention. The other is called compassionate mind training (CMT). CMT consist of a set of practices that work with physiological processes, such as breathing, body posture, facial expressions, voice tone training, and imagery known to be associated with sociality, friendliness and mental well-being (Keltner et al., 2014). In addition, CMT practices focus on conceptualising and engaging with an inner sense of one's own compassionate self- identity, with certain qualities such as kindness, wisdom, strength and commitment. Imagining oneself attending, thinking and behaving from that self-identity 
is a key aspect of CMT practice and taps into one's ability to cultivate and embody the compassionate self qualities into everyday life. So for example in a stressful situation slowing the breath and trying to think through "if I was at my compassionate wisest and strongest how would I like to think, how would I like to act, in this moment" (Gilbert, 2010; Gilbert \& Choden, 2013). In fact, in Tibetan Buddhist traditions the cultivation of one's compassionate self and identity is the essence of Bodhichitta practice and core to developing well-being for self and others and ethics (Dalai Lama, 1995; Jinpa, 2015; Tsering \& McDougall, 2005).

In line with the importance of imagining, thinking and acting (i.e. embodying) from a particular sense of self, there is evidence that practicing imagining one's 'best possible self' is related to increased optimism (Meevissen, Peters, \& Alberts, 2011) and improved coping behaviour (Peters, Flink, Boersma, \& Linton, 2010). Osimo et al. (2015) found that imagining oneself as a virtual Freud (from which one offered oneself counseling) rather than just one's ordinary self resulted in mood improvements. Imagining oneself in different roles and mental states also has an impact on coping. For example, Stopa, Brown and Hirsch (2012) found that practicing positive self-imagery of recalling a time when one felt relaxed and positive was related to higher levels of self-esteem and reduced anxiety in response to anxiety-provoking vignettes (e.g. meeting your partner's parents for the first time). Thus, even though the cultivation and embodiment of a compassionate self-identity in everyday life and in moments of difficulty is core to CMT practices, there is still a lack of studies exploring this ability and its impact on the effects of CMT.

Early studies of CMT have demonstrated effectiveness even with complex psychological difficulties (Beaumont \& Martin, 2013; Braehler, Gumley, Harper, Wallace, Norrie, \& Gilbert, 2013; Gilbert \& Procter, 2006; Mayhew \& Gilbert, 2008). More recent studies using non-clinical populations also found evidence for the effectiveness of CMT. For example, McEwan and Gilbert (2016) showed that a two-week intervention of elements of CMT (soothing rhythm breathing, imagining oneself as a compassionate person, and imagining receiving compassion and care from another person or being) produced increases in self-compassion, and reductions in self-criticism, depression, anxiety and stress that were maintained over six months.

More recently, Matos et al. (2017) conducted a pilot randomized controlled study testing the impact of a two-week CMT intervention on emotional, self-evaluative and psychopathology measures 
and on heart rate variability (HRV) in a nonclinical sample. Participants in the CMT condition attended a psychoeducational group session introducing them to the concepts of compassion and emotion regulation systems, and the CMT practices: soothing rhythm breathing; friendly facial expressions and voice tones; mindfulness; compassionate imagery and focusing. Participants were given a written manual and online access to set of downloadable audio files with guidance for the practices. They were encouraged to practice everyday and to bring compassion into their everyday life, especially when they encountered life difficulties or upsets. Compared to the control group, the CMT group showed significant increases in safe and relaxed positive affect, in self-compassion, compassion for others and sense of compassion from others; and reductions in shame, self-criticism, fears of compassion, and stress. The experimental group also reported significant improvement in HRV, a physiological indicator of adaptive emotion regulation and well-being (Thayer et al., 2012), affiliative emotions and prosociality (Porges, 2007). These findings show that a light-touch CMT intervention was indeed effective in improving several wellbeing indicators. Light-touch interventions are costeffective, time efficient and improve treatment availability access and engagement compared to more intensive approaches. Thus, while CFT targets clinical conditions and requires an intensive faceto-face treatment delivery, an effective brief CMT intervention may be particularly useful to target public health concerns and promote of mental wellbeing among the general population.

To date there have been few studies that have explored the micro skills and micro-processes associated with CMT. In one of the early studies, Gilbert and Procter (2006) designed a diary of change that asked group therapy participants to monitor both self-critical and selfcompassionate/soothing thoughts throughout the week, according to several dimensions: frequency, power, intrusiveness, duration, distress/hostility/use of distraction from (for self-critical thoughts), and soothing/calming/maintenance of (for self-compassionate thoughts). Although diary data were not analysed due to insufficient completion by participants, it was a discussion point in the group therapy. The general sense was that firstly, specific instances of self-criticism were difficult to identify but became clearer as therapy progressed. Second, over time individuals began to notice that they were able to generate and feel more moved and comforted by compassion focusing.

Even though there is growing evidence of the positive effects of CMT interventions on a range of psychological and physiological well-being indicators, it is still unclear how participants' 
practice, subjectively experience and perceive the practices and how these experiences may affect the impact of CMT. Specifically, CMT practice could be quantified in a number of different ways, such as in terms of frequency and duration of formal practices (e.g., soothing breathing, compassionate imagery practices) and embodiment of a compassionate self-identity in daily life (e.g., ability to think, feel and act as one's compassiante self). Another important aspect that has also received little empirical attention is the perception of helpfulness of the practices and its impact of CMT effectiveness. Therefore it is important to explore these micro skills and processes of CMT. For example, is it how often people practice operating from the compassionate self, how helpful they find it and/or the felt experience of compassionate self-states that has most impact on emotional, selfevaluative and well-being indicators?

The aims of this study were therefore threefold. First, we aimed to explore in detail participants feedback on the practice quality of a brief CMT, including the frequency of practice, the perceived helpfulness of practice, and the capacity to act and feel as one's compassionate self in everyday situations and moments of difficulty (i.e., embodiment). Secondly, we explored the associations between the quality of the CMT practices and the three flows of compassion dimensions: compassion for the self; compassion for others and compassion from others. Other measures include impacts on: self-reassurance and self-criticism, shame, compassionate and self-image goals, and different types of positive affect. These outcome variables were chosen because we were interested in exploring whether a CMT intervention would have an impact on: a) participants' level of compassion (i.e., the three flows of compassion and compassionate goals); b) core processes associated with psychopathology that CFT aims to target (i.e., self-criticism and shame); and c) a measure of positive affect and well-being, which is related to the experience of compassion. Third, the study tested the predictive effects of the frequency and perception of helpfulness of the CMT practices, and the embodiment of the compassionate self (comprising several dimensions: frequency, easiness, power, soothing effect, impact and duration, on the ability to receive compassion (from the self and from others) and we need to be compassionate to others.

\section{Method}




\section{Participants}

Participants included 9 (11.7\%) men and 68 (88.3\%) women. Participants' age ranged between 18 and 43 , with a mean age of $23.53(S D=4.22)$. The years of education mean was $15.22(S D=2.33)$. The majority of the sample comprised college students (79.2\%).

\section{Procedure}

The sample recruitment procedures and intervention conditions used in the current study have been described in detail in (Matos et al., 2017). The study was advertised through announcements posted on Coimbra university's campus, and Faculties' mailing lists as a study investigating the effect of meditation on wellbeing indicators. Individuals interested in taking part in the study contacted the research team via email, and were then informed about the procedures of the study and provided their written informed consent. Exclusion criteria were: Major psychiatric or cognitive problems, psychotic or organic illnesses, substance abuse, cardiovascular disease, use of drugs/medications that might affect cardiovascular function, obesity (body mass index $>30 \mathrm{~kg} / \mathrm{m}^{2}$ ), menopause, use of oral contraceptives during the previous 6 months, and pregnancy or childbirth within the last 12 months.

Each participant was randomly assigned to either the compassionate mind training (CMT; $n=$ 63) condition or a wait-list control condition (WLC; $n=54)$. Participants were asked to refrain from a) eating, b) drinking alcohol, tea, or coffee, and c) strenuous exercise 2 hours preceding the scheduled appointment. First, participants came to the laboratory to complete the baseline self-report and physiological (heart rate variability) measures. Participants assigned to the CMT condition were invited to attend a 2-hour group session where they were introduced to the concept of compassion, emotion regulation systems, and the CMT practiced. The researchers clarified any questions participants had regarding the practices. They then provided a written manual outlining the evolutionary theory behind the compassionate mind training, with explanations of emotion regulation and the value of compassion (the manual is available on request). They also provided audio files of the CMT practices for subsequent independent practice. These included: (1) A breathing practice that stimulates the vagal system at around 5-6 breaths per minute (Lin, Tai, \& Fan, 2014); (2) a practice focused on creating facial expressions and friendly voice tones as part of compassion (Gilbert 2000, 2010; Porges, 2007); (3) A practice aimed to develop mindfulness and increase 
attention to one's current mental state; (4) A practice aimed to develop the sense and awareness of one's compassionate self that is based upon feelings of wisdom, strength and commitment to be supportive and helpful to self and others; (5) An imagery practice aimed to develop a compassionate image of another mind that has caring intent towards the self; (6) A practice aimed to develop a compassionate self that has caring intent towards the self and how to use compassion focusing to work with self-criticism and life difficulties (Gilbert 2010; Gilbert \& Choden, 2013).

Participants were encouraged to practice these different exercises over the following two weeks and the importance of doing so for their well-being and research was highlighted. Once they had listened to the instructions on the audio files and understood the practices they were free to use the audio files or not when practising. They were encouraged to bring compassion into their everyday life -- especially when they encountered life difficulties or upsets. At the end of the first week participants completed the qualitative feedback questionnaire at home. After the two-weeks, all participants were invited back to the laboratory to complete the post-test measurements (qualitative feedback questionnaire for week 2, self-report measurements and HRV). Participants received compensation for their participation (15€ voucher).

After these two weeks participants in the WLC were offered the opportunity to complete the entire Compassionate Mind Training protocol and completed the post-intervention assessment two weeks later and the qualitative feedback questionnaire at the end of weeks 1 and 2. The majority of participants (20 out of 37 WLC participants) completed the CMT protocol. The current study was conducted in all participants that completed the CMT intervention and all assessments (CMT $n=56+$ WLC $=20 ; N=77)$. There were no significant differences between the CMT and WLC groups on their baseline measures.

\section{Measures}

Demographics form: Participants were asked to complete a socio-demographic form, which included items regarding gender, age, level of education, height and weight for BMI calculation, and smoking habits ("Are you a smoker?" Yes/No).

Practice diaries: practice diaries were developed based on the original Gilbert and Procter (2006) diaries to be retrospectively filled in each week. The adapted diaries measured: Practice 
frequency ("How often did you practice the exercises during this week?") was measured on a 5-point scale: "never", "1-2", "3-4", "5-6", and "7 or more times per week"; the helpfulness of the compassion practices ("How helpful did you find these practices?") was assessed in a 5-point scale: "Unhelpful", "Not very helpful", "Neither helpful nor unhelpful”, "Quite helpful”, "Very helpful”.

We also developed a set of 10 questions to explore several dimensions regarding the embodiment of the compassionate self in everyday life and in moments of difficulty: Presence of embodiment (“Looking back over the week, can you recall acting or feeling as your compassionate self?") rated as "Yes" or "No"; Embodiment situations ("In which situations did you find yourself acting or feeling as your compassionate self?"), responses to this open-question were grouped thematically; Frequency ("How often did you act as your compassionate self?", "How often did you feel as your compassionate self?") both rated on a 10-point scale ranging from 1 "Never" to 10 "A lot of the time"; Power ("How powerful were your compassionate feelings?") rated on a 10-point scale ranging from 1 "Not powerful at all" to 10 "Very powerful"; Easiness ("How easy was it to act as your compassionate self?", "How easy was it to feel as your compassionate self?") rated on a 10-point scale ranging from 1 "Not easy at all" to 10 "Very easy"; Duration ("How long did your compassionate feelings last?") rated on a 10-point scale ranging from 1 "Fleeting" to 10 "Most of the day"; Soothing effect ("How comforting were your compassionate feelings?") rated on a 10-point scale ranging from 1 "Not comforting at all" to 10 "Very comforting"; Impact of acting as the CS ("How was the impact of your compassionate actions?") rated on a 10-point scale ranging from 1 "Very negative" to 10 "Very positive". A total Embodiment score was computed by summing the dimensions frequency, easiness, power, duration, soothing effect and impact.

Compassionate Attributes and Actions Scales CAAS (Gilbert et al., 2017) Compassion is typically regarded as having two core components 1 . As a sensitivity to the suffering of self and others; 2 . A commitment to try to alleviate and prevent suffering (Gilbert \& Choden, 2013). The CAAS measures different elements of these two psychologies. In regard to the engagement scale, it measures the degree to which individuals are (for example) motivated to engage with suffering, distress tolerance, and empathy. In regard to trying to alleviate and prevent suffering, the scale measures (for example) people's ability to problem solve and behave in ways that are helpful. There are three scales measuring compassion to self, compassion to others, and aware of and experiencing compassion 
from others. Each scale can be analysed in terms of the engagement and action aspects separately or as a single factor. Here we will use each of the three orientations (compassion for self, compassion for others and sensitivity to the compassion from others) as a single factor.

Compassionate and Self-Image Goals (CSIG; Crocker \& Canevello, 2008; Portuguese Version by Duarte, Pinto-Gouveia, \& Lopes, unpublished manuscript). The CSIG is a 13-item scale that uses a 5point rating scale $(1=$ not at all to $5=$ always $)$ to assess the extent to which subjects hold more compassionate or self-image goals regarding their friendships. There are 7 compassionate goals' items (e.g., 'have compassion for others' mistakes and weaknesses') and 6 self-image goals' items (e.g., 'avoid the possibility of being wrong'). All items begin with: "In the past two weeks, in the area of friendship, how much did you want to or try to..." In the original study Cronbach's Alpha was .83 for Self-Image Goals and .90 for Compassionate Goals.

Types of Positive Affect Scale (Gilbert et al., 2008) This scale was developed to measure the degree to which people experience different types of positive emotions. Respondents are asked to rate 18 'feeling' words on a 5 -point scale to indicate how characteristic it is of them $(0=$ 'not characteristic of me' to $4=$ 'very characteristic of me'). Factor analysis revealed three factors or subscales, these are: Activated Positive Affect (e.g., "excited", "dynamic", "active"); Relaxed Positive Affect (e.g., "relaxed", "calm", "peaceful") and Safeness/contentment Positive Affect (e.g., "safe", "secure", "warm"). The scale showed good psychometric properties with Cronbach alphas of .83 for Activating Positive Affect and Relaxed Positive Affect, and .73 for Safeness/contentment Positive Affect.

Forms of Self Criticising/Attacking and Self Reassuring Scale (FSCRS) (FSCRS; Gilbert, Clarke, Kempel, Miles, \& Irons, 2004). This 22 -item scale measures people's critical and self-reassuring selfevaluative responses to setbacks or disappointments. Participants rate on a 5-point scale (ranging from $0=$ not at all like me to $4=$ extremely like me) how they might typically think and react when things go wrong for them. The scale measures two forms of self-criticism: Inadequate self, which focuses on a sense of personal inadequacy (e.g. "I am easily disappointed with myself") and Hated self, which measures the desire to hurt or persecute the self (e.g. "I have become so angry with myself that I want to hurt or injure myself"). In this study a total of Self-criticism was used by summing Inadequate self and Hated self scores. In addition, the scale measures self-reassuring and 
supportiveness when things go wrong (e.g. "I am able to care and look after myself"). The scale had Cronbach's alphas of .90 for inadequate self, .86 for hated self and .86 for reassured self (FSCRS; Gilbert et al., 2004). A number of replication studies have supported the reliability of the scale (e.g, Baião, Gilbert, McEwan, \& Carvalho, 2014; Castilho, Pinto-Gouveia, \& Duarte, 2013; Kupeli, Chilcot, Schmidt, Campbell, \& Troop, 2013).

Other as Shamer scale (Goss, Gilbert, \& Allan, 1994). This scale was developed from Cook's (1994) Internalized Shame Scale by Allan, Gilbert and Goss (1994) and Goss et al. (1994). It assesses global judgments of how people think others see them (e.g. "I think other people see me as inadequate") thus focusing on external shame rather than internalized shame. The scale consists of 18 descriptions of feelings or experiences and respondents indicate the frequency on a 5-point likert scale from 0 (never) to 4 (almost always). Goss et al. (1994) found the scale to have a good Cronbach alpha of 0.92 . Because the scale asks about how often people think this way, it measures thought frequency.

\section{Data analyses}

The analyses were carried out using SPSS Version 22 and AMOS version 21. Data was checked for normality of distribution and outliers. There were no extreme outliers. No variable had indicators of severe violations to the normal distribution (Sk <| $3 \mid$ and $\mathrm{Ku}<|10|$; Kline, 2005).

Descriptive statistics were conducted to explore the frequency of situations where participants acted or felt as their compassionate self at the end of each week of practice. Descriptive statistics (mean and standard deviation) were also performed to analyze qualitative feedback data (composite indices of week 1 and 2 ) and the other self-report variables at post-intervention. The baseline compassion scales scores were included as control variables in the path analyses, and therefore descriptive statistics are also presented for these variables. Correlation analyses were calculated to explore the associations among practice frequency, helpfulness, embodiment total and its six dimensions (composite indices of week 1 and 2) and between these variables and the other self-report variables at post-intervention.

Structural equation models were conducted to test the predictive effect of the qualitative feedback variables on the three flow of compassion variables - compassion for the self, compassion 
for others and compassion from others - at post-test, while controlling for baseline values of these variables. In these models, a measurement model was computed to estimate the latent variable Embodiment measured by six observed variables, derived from the CFT theoretical model. A Maximum Likelihood method was used to evaluate the regression coefficients significance and to compute fit statistics. Model fit was assessed by the following model fit indicators: $\chi^{2} / \mathrm{df}$, Tucker Lewis Index (TLI), Comparative Fit Index (CFI), Root-Mean Square Error of Approximation (RMSEA) and Standard Root-Mean Square Error of Approximation (SRMR; Kline, 2005).

\section{Results}

Descriptive data regarding practice frequency showed that $28.2 \%(n=24)$ of participants practiced the exercises 1 or 2 times a week, 38.8\% (n=33) 3 or 4 times, $8.2 \%(n=4) 5$ or 6 times and $2.4 \%(n=2)$ every day or more than once per day. No participant responded "never" to the practice frequency question. So nearly $50 \%$ had regular practice. When asked about the helpfulness of the CMT practices the majority of participants $(49.4 \%, n=42)$ considered them as quite helpful and $30.6 \%(n=26)$ as very helpful. Only one participant thought the exercises weren't very helpful and 6 (7.1\%) that they were neither helpful nor unhelpful. As to whether they were able to act or feel as their compassionate self over the week, $90.6 \%$ of participants responded 'Yes' and only one said 'No'. When asked about the situations in which they found themselves acting and feeling as their compassionate self, in general the most reported situations during the study period were coping with difficult emotions, sensitivity to and helpfulness to others, unspecific interpersonal situations and work-related stress situations (see Table 1).

Insert Table 1 approximately here

Regarding the other embodiment dimensions, in rating scales ranging from 1 to 10 , frequency had a mean $(M)$ score of $6.50(S D=1.49)$, easiness $M=6.10(S D=1.69)$, duration $M=6.29$ 
$(S D=1.57)$, power of the compassionate feelings $M=6.38(S D=1.48)$, soothing effect $M=7.46(S D=$

1.77), impact of compassionate actions $M=7.40(S D=1.58)$.

Descriptive statistics for the self-report variables are presented in Table 2.

Insert Table 2 approximately here

Correlation coefficients are presented in Table 3. Results show that practice frequency over the two-week period was positively associated with the frequency and easiness of embodiment of the compassionate self (CS). Participants' perception of the helpfulness of the practices was related to greater embodiment of the CS in everyday life, as well as to the frequency, the power, the soothing effect and the impact of feeling and acting as the CS. The perception of the helpfulness of the practices was also positively associated with compassion for the self, receiving compassion from others, and with self-reassurance; with relaxed and safe positive affect; and was negatively associated with self-criticism. Regarding the embodiment of the CS in everyday life and in moments of difficulty results indicated strong positive associations between the dimensions frequency, easiness, power, duration, soothing effect and impact of acting and feeling as the CS. The total of embodiment was strongly correlated with compassionate for self and positively associated with compassion for others and from others, reassured self, compassionate goals and safe and relaxed positive affect. The embodiment dimensions were also positively associated with compassion for the self, compassion for others (with the exception of the easiness dimension), self-reassurance, and compassionate goals. The power, soothing effect and the impact of acting and feeling as the CS were also positively associated with receiving compassion from others and with safe positive affect. Furthermore, the duration of the compassionate feelings was positively associated with relaxed positive affect. Practice frequency, helpfulness and embodiment was not significantly associated with shame.

Insert Table 3 approximately here 
To test the predictive effect of the Helpfulness of the practices and the Embodiment of the compassionate self on compassion for the self, compassion for others and compassion from others, three separate path analyses were calculated. A latent Embodiment variable of the different dimensions - frequency, easiness, power, soothing effect, impact, and duration - was estimated in the model. Practice frequency was not included in the models as it was not significantly associated with the other variables of interest. Results for the three models are reported in Figure 1.

Compassion for the self. The model accounted for $46 \%$ of Compassion for the self variance. The model revealed an excellent model fit $(\mathrm{CMIN} / \mathrm{DF}=1.67 ; \mathrm{CFI}=.96 ; \mathrm{TLI}=.94 ; \mathrm{RMSEA}=.096, \mathrm{Cl}$ $[.036 ; .148], p=.088 ;$ SRMR $=.053)$. Results indicated that the different Embodiment dimensions presented high standardized regression weights on the latent Embodiment variable (ranging from .67 to .92). Embodiment presented a direct effect of .47 ( $b=5.74 ; S E=1.40 ; z=4.11, p<.001)$. Helpfulness of the practice also showed a significant direct effect of $.23(b=4.70 ; S E=1.87 ; z=2.52$, $p=.012)$

Compassion for others. Even though the model revealed a good fit $(\mathrm{CMIN} / \mathrm{DF}=1.68 ; \mathrm{CFI}=$ $.96 ; \mathrm{TLI}=.94 ; \mathrm{RMSEA}=.097, \mathrm{Cl}[.038 ; .149], p=.082 ; \mathrm{SRMR}=.056)$, results showed that the direct paths between Embodiment $(p=.065)$ and Helpfulness $(p=.937)$ and compassion for others at postintervention were non-significant.

Compassion from others. The model accounted for $46 \%$ of Compassion from others variance. The model revealed an excellent model fit $(\mathrm{CMIN} / \mathrm{DF}=1.85 ; \mathrm{CFI}=.95 ; \mathrm{TLI}=.92 ; \mathrm{RMSEA}=.110, \mathrm{Cl}$ [.056; .160], $p=.038 ;$ SRMR = .062). Results showed Embodiment presented a direct effect of $.23(b=$ 3.09; $S E=1.34 ; z=2.31, p=.021)$. Helpfulness of the practice presented a marginally significant direct effect of $.18(b=3.85 ; S E=1.99 ; z=1.94, p=.052)$.

\section{Discussion}

This study explored some of the micro processes associated with CMT, in particular how people actually practice and experience the compassion cultivation practices, and is part of a larger project looking at the effectiveness of compassionate mind training in a nonclinical population (Matos et al., 2017). In the current study the majority of people practiced compassionate imagery and imagining compassionate self engagement. Around $50 \%$ of people practiced three or more times a 
week. These results extend previous research (McEwan \& Gilbert, 2016) showing that most participants enrolled in a brief CMT online intervention engaged in frequent practice of compassionate imagery exercises.

Results showed that the frequency with which participants practiced CMT during the two weeks (practice frequency) was related to how easy and often participants reported they were able to feel and act as if they were their compassionate self. Interestingly, practice frequency was not correlated with the other embodiment dimensions. In addition, the frequency of the practice was not associated with compassion (for self, for others and from others), reassured self, compassionate goals, safe/relaxed positive affect, shame and self-criticism at post-intervention. These findings contrast previous research by Jazaieri et al. (2015) showing that the frequency of compassion meditation practice during a 9-week compassion meditation program had beneficial effects on selfreport caring behaviors for oneself and others. Nonetheless, to the best of our knowledge there are no studies that have looked on the association between practice frequency and helpfulness and compassion intervention outcomes, such as compassion, positive affect and psychological adjustment.

In regard to practice helpfulness, most participants considered the CMT practices as quite or very helpful. This is in line with McEwan and Gilbert (2016) who reported that participants qualitative feedback was that a brief CMT online intervention helped them to feel more calm and content in themselves and with others. Furthermore, participants' perception of how helpful the CMT practices were for them was related to greater ability to embody the CS in everyday life. In particular, practice helpfulness was related to how frequent, powerful, soothing and impactful it was to feel and act as their CS. In contrast to practice frequency, the perceived helpfulness of the practices was linked to increased capacity to direct compassion towards the self and to experience others as compassionate. In addition, at post intervention, the perceived helpfulness was related to being self-reassuring in times of difficulty, to feeling relaxed and safe, and to decreased self-criticism. It is possible that the perception of helpfulness of the exercises was supported by the evolutionary insights into the nature of human's evolved brain, and how compassion focusing can help with some of the difficulties that arise from its complexity. Therefore, an interesting question for future studies is to explore the helpfulness of the practices with and without the psychoeducational element in a CMT intervention. 
Regarding the embodiment of the CS, the vast majority (90.6\%) of participants reported they were able to act or feel as their compassionate self in everyday situations or moments of difficulty during the study period. Also, participants presented high mean scores in terms of frequency, easiness, power and duration of embodiment of the CS, and scored even higher in terms of the soothing effect and impact of their compassionate feelings and actions. This may support the value of using embodied acting techinques, where individuals are instructed to first imagining themselves at their compassionate best with breathing techniques, and then approach difficult life situations from that sense of self, to encourage the cultivation of the compassionate self (Gilbert, 2010; Kirby \& Gilbert, 2017).

Rather than just practicing the compassionate self and compassionate mind focusing as a separate specific task, participants were advised to bring that mindset into everyday life and use it for coping with various situations. This study reveals that the situations in which participants were able to embody the CS were mostly related to coping with difficult emotions, sensitivity to and helpfulness to others, unspecific interpersonal situations and work-related stress situations. Therefore, participants reported they were able to use CS abilities to cope with various situations in their lives, in particular to regulate difficult emotions and in challenging interpersonal situations. These findings suggest that cultivating the compassionate self through CMT insight and practices seems to promote a greater ability to feel and act compassionately towards the self and also to direct this flow of compassion towards others in everyday life situations. These findings fit in with other studies that have explored the impact of self-focusing practices, such as imagine one's best possible self (Meevissen et al., 2011; Peters et al, 2010) and imagine advising oneself as a virtual Freud (Osimo et al., 2015).

The embodiment of the compassionate self in everyday life and in moments of difficulty was related to being more compassionate and reassuring towards oneself, to experiencing others as more compassionate, to being more compassionate towards others, and to having more compassionate goals in one's relationships. Results also revealed that when people embody their compassionate self that is associated with positive emotions. In particular, the power, soothing effect and impact of acting and feeling as the compassionate self were associated with feelings of safeness and contentment, while the duration of the compassionate feelings was linked to increased feelings of calmness and relaxation. Of note, the capacity to embody the CS was not related to threat-based 
emotions and processes, such as self-criticism and shame, and self-image goals. Correlational findings also demonstrated that the six dimensions of embodiment of the CS - frequency, easiness, power, duration, soothing effect and impact - were positively and strongly inter-correlated. These results are in line with CMT theoretical approach suggesting that the engagement and embodiment of an inner sense of one's compassionate self-identity is a core feauture of this training and has a crucial role on the impact of the intervention on compassion and well-being (Gilbert, 2010; Gilbert \& Choden, 2013; Jinpa, 2015).

Interestingly, when looking at the correlations between the practice quality variables and the self-report variables, results reveal that it is the perception of helpfulness of the CMT practices and the embodiment of the compassionate self dimensions, and not the frequency of the practice, that are associated with post-intervention compassion related outcomes and positive affect.

Therefore, path analyses were conducted to better understand the impact of the perceived helpfulness of CMT practices and the embodiment of the CS on the development of the three dimensions of flow of compassion. First, results confirmed that the six dimensions of frequency, easiness, power, duration, soothing effect and impact of embodiment of the $\mathrm{CS}$, underlie the latent construct of embodiment of the CS, which corroborates the CFT theoretical framework on the importance of cultivating and engaging with a compassionate self-identity, by thinking, feeling and acting as the compassionate self in everyday life and, in particular, in moments of stress and difficulty. Second, the embodiment of the CS and perceived helpfulness of the practices predicted changes in both compassion for the self and the experience of receiving compassion from others, and explain almost half of their variance. However, when controlling for baseline values, compassion to others at post intervention was not predicted by neither the embodiment of the CS nor the perceived helpfulness of the practices.

Our results are consistent with the findings from Gilbert et al. (2011) that different processes underpin being the recipient of compassion (for self and from others) in contrast to offering compassion to others. Fears of being compassionate towards oneself and being open to the compassion from others have weak correlations with the fear of being compassion to others. In other words, one can be fearful of being the recipient of compassion but not of giving it. Although developing the compassionate self would appear to be related to becoming compassionate towards 
others, in our studies (see also Matos et al., 2017) when individuals become their compassionate self they become more self-compassionate and experience the world more compassionately. This links with the notion that compassion is in fact a self-identity system that, when cultivated and/or activated, organizes one's mind and how one experiences oneself and the world (Gilbert, 2010, 2014; Gilbert \& Choden, 2013). In general, this study provides evidence that perceiving compassion cultivation practices as helpful and being able to embody the compassionate self in everyday life and in moments of difficulty, are key to foster self-compassion and the experience of receiving compassion from others, as well as to promote feelings of safeness, contentment and calmness.

The findings from our study may open new avenues for future research. Our results indicate it might be important to further investigate the importance of helping people to embody the different dimensions of the compassionate self, and not just how often they practice the compassion training exercises. These data also suggests that it might be relevant to investigate the impact of working with both the facilitators and the inibitors of the embodiment of the compassionate self. For example, the perception of helpfulness of the CMT practices is related to the embodiment and development of the compassionate self. Therefore the psychoeducational component of CMT and CFT (Gilbert, 2010) could be highlighted and worked with as it might be an fundamental way to foster the perceived helpfulness of compassion cultivation. Therefore, along with encouraging a continued practice of CMT exercises, the importance and helpfulness of embodying and cultivating the compassionate self in participants' everyday life should be highlighted.

\section{Limitations}

Even though this is the first study to explore, using an RCT design, the role of practice frequency, helpfulness and embodiment of the compassionate self in promoting the effectiveness of a CMT intervention, some methodological limitations should be considered in interpreting these findings. A limitation of the present study is that the impact of each individual component of CMT, such as the psychoeducation, the breathing and the compassion imagery and focusing practices, were not studied separetely in detail. Future research should seek to investigate the separate effects of each of these CMT components on compassion, positive affect and psychopathology outcomes. In addition, this study used a non-clinical population and therefore future studies should seek to replicate it using a clinical sample. Also the sample was mostly female and in the future, studies could 
explore potential gender differences in these CMT micro skills and processes. These findings are reliant on self-report data and may thus be subject to response bias, and there was no follow-up measurement. Future studies should extend this research and implement other assessment methodology (e.g., experience sampling, observation of actual behaviours, qualitative interview) and assess the stability of the findings over time.

\section{Compliance with Ethical Standards}

Funding: This study was funded by the first author (MM) PostDoctoral grant (SFRH/BPD/84185/2012), sponsored by the Portuguese Foundation for Science and Technology (FCT).

This work was also part funded by the Compassionate Mind Foundation charity (www.compassionatemind.co.uk).

Ethical approval: All procedures performed in studies involving human participants were in accordance with the ethical standards of the Faculty of Psychology and Educational Sciences of the University of Coimbra, and with the 1964 Helsinki declaration and its later amendments or comparable ethical standards.

Informed consent: Informed consent was obtained from all individual participants included in the study.

Conflict of Interest: The authors declare that they have no conflict of interest.

\section{Author Contributions}

MM designed and executed the study, performed the data analyses, and wrote the paper. JD assisted with the design and implementation of the study, data analyses and writing of the paper. CD assisted with the design and implementation of the study, data analyses and writing of the paper. PG collaborated with the design of the study, discussion of results and writing of the paper. JPG collaborated with the design the study, data analyses and discussion of results. 


\section{References}

Allan, S., Gilbert, P. \& Goss, K. (1994). An exploration of shame measures-II: Psychopathology. Personality and Individual Differences, 17(5), 719-722.

Beaumont, E., \& Martin, C. J. H. (2013). Using compassionate mind training as a resource in EMDR: A case study. Journal of EMDR Practice and Research, 7(4), 186-199. doi:10.1891/19333196.7.4.186

Braehler, C., Gumley, A., Harper, J., Wallace, S., Norrie, J., \& Gilbert, P. (2013). Exploring change processes in compassion focused therapy in psychosis: Results of a feasibility randomized controlled trial. British Journal of Clinical Psychology, 52, 199-214.

Crocker, J. \& Canevello, A. (2008). Creating and undermining social support in communal relationships: The role of compassionate and self-image goals. Journal of Personality and Social Psychology, 95, 555-575.

Cook, D. R. (1994, 2001). Internalized shame scale: Technical manual. North Tonawanda, NY: Multi-Health Systems, Inc.

Dalai Lama. (1995). The Power of Compassion. India: HarperCollins.

Fredrickson, B. L., Cohn, M. A., Coffey, K. A., Pek, J., \& Finkel, S. M. (2008). Open hearts build lives: Positive emotions, induced through loving-kindness meditation, build consequential personal resources. Journal of Personality and Social Psychology, 95(5), 1045-1062.

Gilbert, P. (2000). Social mentalities: Internal 'social' conflicts and the role of inner warmth and compassion in cognitive therapy. In, P. Gilbert \& Bailey K.G (eds.) Genes on the Couch: Explorations in Evolutionary Psychotherapy (p.118-150). Hove: Brenner-Routledge.

Gilbert, P. (2010). Compassion focused therapy: The CBT distinctive features series. London: Routledge.

Gilbert, P. (2014). The origins and nature of compassion focused therapy. British Journal of Clinical Psychology, 53, 6-41. doi: 10.1111/bjc.12043.

Gilbert, P., \& Choden. (2013). Mindful compassion. London, UK: Constable-Robinson.

Gilbert, P., Clarke, M., Kempel, S. Miles, J. N. V. \& Irons, C. (2004). Criticizing and reassuring oneself: An exploration of forms style and reasons in female students. British Journal of Clinical Psychology, 43, 31-50. doi:0.1348/014466504772812959. 
Gilbert, P., McEwan, K., Matos, M., \& Rivis, A. (2011). Fears of Compassion: Development of three self-report measures. Psychology and Psychotherapy: Theory, research and practice, 84, 239255. doi: $10.1348 / 147608310 \times 526511$

Gilbert, P., McEwan, K., Mitra, R., Franks, L., Richter, A., \& Rockliff, H. (2008). Feeling safe and content: A specific affect regulation system? Relationship to depression, anxiety, stress, and self-criticism. The Journal of Positive Psychology, 3, 182-191.

Gilbert, P. \& Procter, S. (2006). Compassionate mind training for people with high shame and selfcriticism: overview and pilot study of a group therapy approach. Clinical Psychology and Psychotherapy, 13, 353-379. doi: 10.1002/cpp.507

Goss, K., Gilbert, P. \& Allan, S. (1994) An exploration of shame measures-I: The Other As Shamer Scale. Personality and Individual Differences, 17(5), 713-717.

Hofmann, S. G., Grossman, P., \& Hinton D. E. (2011). Loving-kindness and compassion meditation: Potential for psychological intervention. Clinical Psychology Review, 13, 1126-1132. doi:10.1016/j.cpr.2011.07.003

Jazaieri, H., Jinpa, G. T., McGonigal, K., Rosenberg, E., Finkelstein, J., Simon-Thomas, E., \& Goldin, P. R. (2013). Enhancing compassion: A randomized controlled trial of a compassion cultivation training program. Journal of Happiness Studies, 14, 1113-1126. doi: 10.1007/s10902-0129373-z.

Jinpa, T. (2015). A Fearless Heart. Why Compassion is the Key to Greater Well-Being. London: Little Brown

Keltner, D., Kogan, A., Piff, P. K., \& Saturn, S. R. (2014). The sociocultural appraisals, values, and emotions (SAVE) framework of prosociality: Core processes from gene to meme. The Annual Review of Psychology, 65, 425-460. doi: 10.1146/annurev-psych-010213-115054.

Kirby, J.N. (2016). Compassion interventions: The programs, the evidence, and implications for research and practice. Psychology and Psychotherapy: Theory, Research, and Practice. Advance online publication. doi: 10.1111/papt.12104

Kirby, J., Doty, J., Petrocchi, N. \& Gilbert P (2017). The Current and Future Role of Heart Rate Variability for Assessing and Training Compassion. Frontiers Public Health 5, 40. doi: 10.3389/fpubh.2017.00040 
Kirby, J. \& Gilbert, P. (2017). The emergence of the Compassion Focused Therapies. In: P. Gilbert (Ed).

Compassion: Concepts, Research and Applications (p.258-285). London: Routledge

Klimecki, O.M., Leiberg, S., Lamm, C \& Singer, T. (2012). Functional neural plasticity and associated changes in positive affect after compassion training. Cerebral Cortex, advance publication (June 1st) doi:10.1093/cercor/bhs142

Kline, R. (2005). Principles and practice of structural equation modelling (2th ed.). New York : The Guilford Press.

Leaviss, J., \& Uttley, L. (2014). Psychotherapeutic benefits of compassion focused therapy: An early systematic review. Psychological Medicine, 45, 927-945. DOI:10.1017/S0033291714002141.

Lin ,M., Tai, L.Y, \& Fan, S.Y. (2014) Breathing at a rate of 5.5 breaths per minute with equal inhalation-to-exhalation ratio increases heart rate variability International Journal of Psychophysiology, 91 206-11 http://www.ncbi.nlm.nih.gov/pubmed/24380741\#

Matos, M., Duarte, C., Duarte, J., Pinto-Gouveia, J., Petrocchi, N., Basran, J. \& Gilbert, P. (2017). Psychological and physiological effects of compassionate mind training: A pilot randomized controlled study. Mindfulness, 8 (6), 1699-1712. doi. 10.1007/s12671-017-0745-7

Mayhew, S., \& Gilbert, P. (2008). Compassionate mind training with people who hear malevolent voices: A case series report. Clinical Psychology and Psychotherapy, 15, 113-138.

McEwan, K., \& Gilbert, P. (2015). A pilot feasibility study exploring the practising of compassionate imagery exercises in a nonclinical population. Psychology and Psychotherapy: Theory, Research and Practice, 89, 239-243. doi:10.1111/papt.12078

Meevissen, Y.M.C, Peters, M.L \& Alberts, H.J.E.M. (2011). Become more optimistic by imagining a best possible self: Effects of a two week intervention. Journal of Behavior Therapy and Experimental Psychiatry, 42, 371-378.

Osimo, S. A., Pizarro, R., Spanlang, B., \& Slater, M. (2015). Conversations between self and self as Sigmund Freud-A virtual body ownership paradigm for self counselling. Nature Scientific Reports, 5, 13899. DOI: 10.1038/srep13899

Pace, T. W. W., Negi, L. T., Adame, D. D., Cole, S. P., Sivilli, T. I., Brown, T. D., ... Raison, C. L. (2009). Effect of compassion meditation on neuroendocrine, innate immune and behavioral 
responses to psychosocial stress. Psychoneuroendocrinology, 34, 87-98.

doi:10.1016/j.psyneuen.2008.08.011

Peters, M. L., Flink, I.K., Boersma, K., Linton, S.J. (2010). Manipulating optimism: Can imagining a best possible self be used to increase positive future expectancies? The Journal of Positive Psychology, 5, 204-211

Porges, S. W. (2007). The polyvagal perspective. Biological Psychology, 74, 116-143.

Ricard, M. (2015). Altruism. The Power of Compassion to Change Itself and the World. London Atlantic Books

Schanche, E., Stiles, T. C., McCullough, L., Svartberg, M., \& Nielsen, G. H. (2011).

The relationship between activating affects, inhibitory affects, and self-compassion in patients with cluster C personality disorders. Psychotherapy: Theory, Research, Practice, Training, 48, 293-303. doi: 10.1037/a0022012

Singer, T., \& Bolz, M. (Eds.) (2012). Compassion: Bridging practice and science. http://www.compassion-training.org/

Stopa, L., Brown, M. A., \& Hirsch, C. R. (2012). The effects of repeated imagery practice on selfconcept, anxiety and performance in socially anxious participants. Journal of Experimental Psychopathology, 3(2), 223-242.

Thayer, J., Åhs, F., Fredrikson, M., Sollers, J., and Wager, T (2012). A meta-analysis of heart rate variability and neuroimaging studies: implications for heart rate variability as a marker of stress and health. Neuroscience and Biobehavioral Review 36, 747-756. doi: 10.1016/j.neubiorev.2011.11.009

Tsering, T., \& McDougall, G. (2005). The Four Noble Truths: The Foundation of Buddhist Thought (Vol. 1). Simon and Schuster.

Weng, H. Y., Fox, A. S., Shackman, A. J., Stodola, D. E., Caldwell, J. Z. K., Olson, M. C., ... Davidson, R. J. (2013). Compassion training alters altruism and neural responses to suffering. Psychological Science, 24(7), 1171-1180. doi:10.1177/0956797612469537 


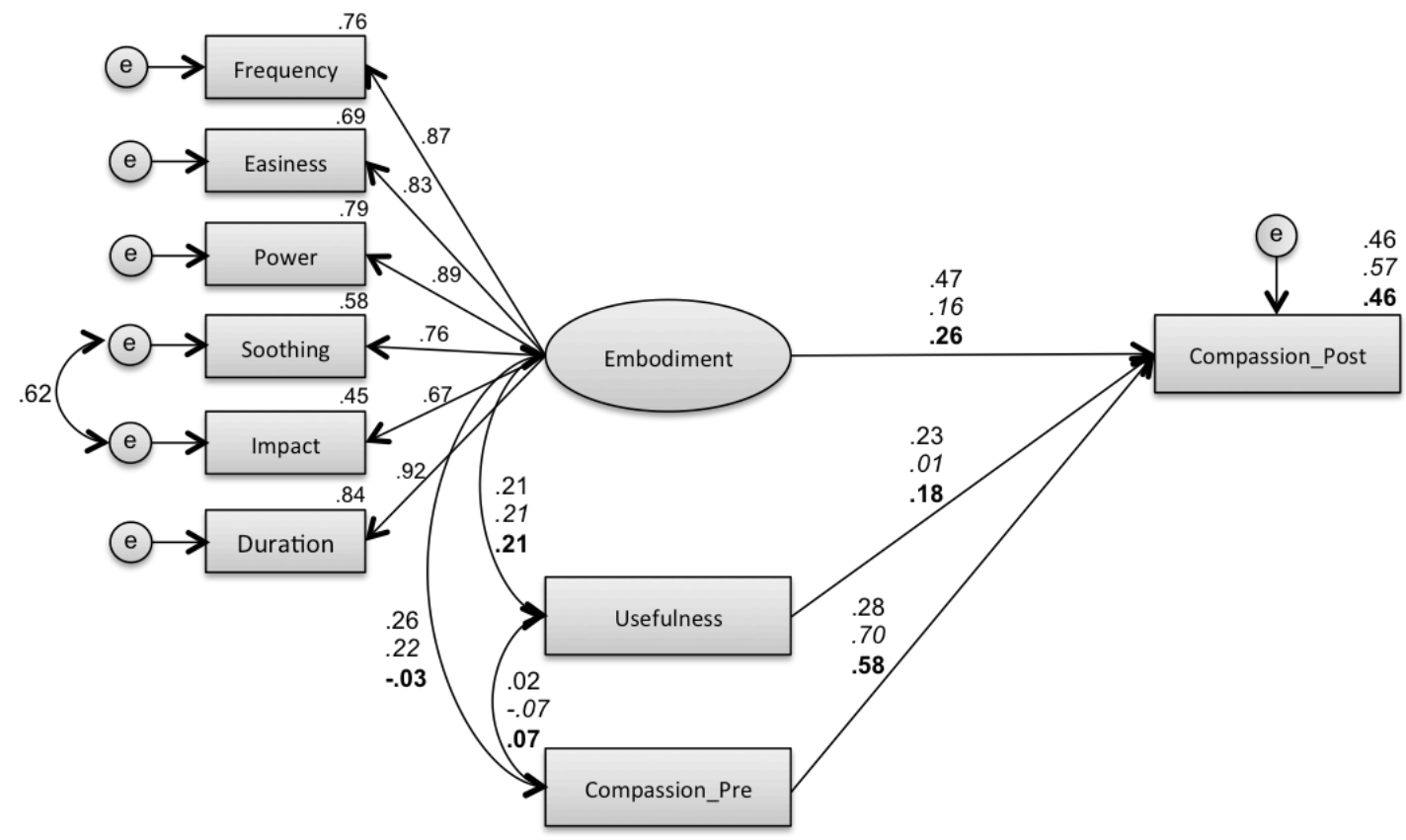

Figure 1. Results of three path analyses showing predictive effect of Helpfulness and Embodiment of the Compassionate Self on Compassion for the self (presented in standard typing), compassion for others (presented in italics) and compassion from others (presented in bold), while controlling for baseline values of these variables 
Table 1.

Descriptive statistics of the frequency of situations in which participants reported acting/feeling as the compassionate self during the two weeks of the CMT

\begin{tabular}{|c|c|c|c|c|}
\hline & \multicolumn{2}{|c|}{ Week 1} & \multicolumn{2}{|c|}{ Week 2} \\
\hline & Frequency & Percent & Frequency & Percent \\
\hline $\begin{array}{l}\text { Unspecific } \\
\text { everyday situations }\end{array}$ & 9 & 12.9 & 5 & 7.0 \\
\hline $\begin{array}{l}\text { Coping with difficult } \\
\text { emotions }\end{array}$ & 19 & 27.1 & 25 & 35.2 \\
\hline Interpersonal conflict & 8 & 11.4 & 6 & 8.5 \\
\hline $\begin{array}{l}\text { Sensitivity to and } \\
\text { helpfulness to others }\end{array}$ & 12 & 17.1 & 6 & 8.5 \\
\hline $\begin{array}{l}\text { Unspecific interpersonal } \\
\text { situations }\end{array}$ & 11 & 15.7 & 12 & 16.9 \\
\hline $\begin{array}{l}\text { Unspecific interpersonal } \\
\text { situations }\end{array}$ & 0 & 0 & 4 & 5.6 \\
\hline $\begin{array}{l}\text { Major negative life } \\
\text { events }\end{array}$ & 2 & 2.9 & 1 & 1.4 \\
\hline Self-criticism & 4 & 5.7 & 2 & 2.8 \\
\hline Work-related stress & 5 & 7.1 & 10 & 14.1 \\
\hline
\end{tabular}


Table 2.

Descriptive Statistics of the Variables in Study

\begin{tabular}{lcc}
\hline & $M$ & $S D$ \\
\hline Compassion for Self_Pre & 65.86 & 12.20 \\
Compassion to Others_Pre & 78.33 & 10.65 \\
Compassion From Others_Pre & 66.39 & 14.25 \\
Compassion for Self_Post & 73.55 & 13.01 \\
Compassion to Others_Post & 81.86 & 9.94 \\
Compassion From Others_Post & 72.55 & 13.82 \\
Reassured Self & 22.46 & 5.31 \\
Self-Criticism & 14.80 & 8.25 \\
Compassionate Goals & 27.96 & 4.39 \\
Self-Image Goals & 16.55 & 5.00 \\
Relaxed Positive Affect & 2.60 & 0.70 \\
Safe Positive Affect & 2.68 & 0.71 \\
Practice Frequency & 1.72 & 0.71 \\
Practice Helpfulness & 3.11 & 0.65 \\
Frequency of embodiment & 6.50 & 1.49 \\
Easiness of embodiment & 6.10 & 1.69 \\
Power of embodiment & 6.38 & 1.48 \\
Soothing effect of embodiment & 7.46 & 1.66 \\
Impact of embodiment & 7.40 & 1.58 \\
Duration of embodiment & 6.29 & 1.57 \\
\hline
\end{tabular}

